\title{
Influence of Mobile Phase Composition and Temperature on the Retention Behavior of Selected Test Substances in Diol-type Column
}

\author{
Wojciech Zapała ${ }^{*}$, Piotr Ziobrowski, Lidia Zapała, Marcin Chutkowski \\ Department of Chemical and Process Engineering, Chemical Faculty, Rzeszów University of Technology, Powstańców Warszawy 6, $35-$ \\ 959 Rzeszów, Poland \\ Email: ichwz@prz.edu.pl
}

\begin{abstract}
In this work the effect of mobile phase composition and temperature on the retention behavior of several test compounds (including non-polar and polar substances) on diol-Acclaim Mixed Mode HILIC-1 column in methanol-water systems with high (RPLC mode) and low (HILIC mode) water content was analyzed. In the studied systems both linear, slightly curved and curved van't Hoff plots were observed. This indicates, that depending on solute physicochemical properties and mobile phase composition, both single and more complex retention mechanism(s) may influence the retention behavior of tested substances. The possibilities of different retention mechanisms existence (e.g. connected with adsorbent surface heterogeneity, hydrophobic and hydrogen bond interactions) in the analyzed systems have been also discussed.

Keywords: diol stationary phase, mobile phase composition, retention, van’t Hoff plot
\end{abstract}

\section{Abbreviations}

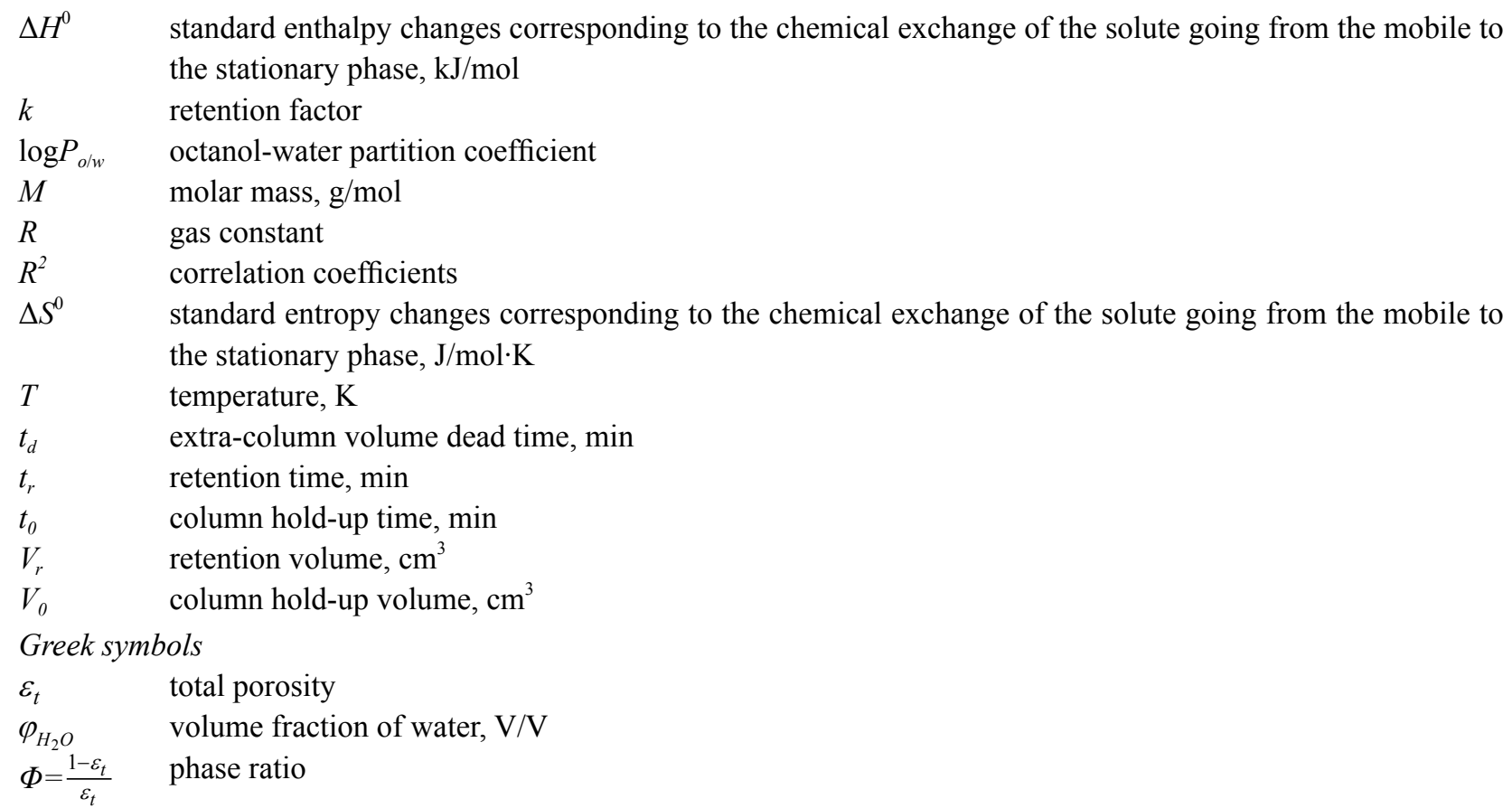




\section{Introduction}

Increased interest in using hydrophilic interaction chromatography (HILIC) based on polar stationary phases combined with partly aqueous eluents (around $2-40 \%$ water) containing different organic solvents, e.g. acetonitrile or alcohols has been recently observed ${ }^{[1,2]}$. The list of useful HILIC stationary phases for various applications is very long and includes any silica-based or organic-polymer polar stationary phases ${ }^{[1]}$. Chemically bonded diol stationary phases are also used in HILIC. However, diol columns are less popular compared to other HILIC packings ${ }^{[1]}$. In this respect, it seems obvious that the potential of diol-type stationary phases is not yet adequately explored both from practical and theoretical point of view. These adsorbents show high polarity and hydrogen bonding properties, like the bare silica, and do not contain ionizable groups other than non-reacted residual silanols that can be partially blocked by a silylating reagent to avoid irreversible adsorption of polar compounds. Diol-silica columns have been mainly used for separations of vitamins, proteins, low molecular phenolic compounds and flavonoids ${ }^{[1]}$. The overview of applications of different diol stationary phases can be found in e.g. [1]. The diol-type Acclaim Mixed Mode HILIC-1 column is relatively new (first described in 2008 year $\left.^{[3]}\right)$. The bonding of this chromatographic material contains both hydrophilic functionalities, i.e. diol groups and also hydrophobic functionalities, i.e. C10 alkyl chain. Note that conventional HILIC diol phases have a short (usually three carbon) alkyl chain that merely serves as a linker between the terminal polar group and the attachment point on the silica surface. Therefore, this HILIC phase is termed to be a multi modal packing material which means it can be operated either in HILIC mode (in highly organic conditions) or in reversed phase liquid chromatography (RPLC) mode (in highly aqueous conditions) and provides great potential for separation of a wide range of polar and non-polar molecules ${ }^{[3]}$. According to the manufacturer, the Acclaim column exhibits greatly increased hydrophobic retention for neutral, non-polar molecules compared to a conventional diol column and works in reversed-phase mode when the mobile phase contains less than about $70 \%$ organic solvent by concentration. At the same time the column works in HILIC mode when the mobile phase concentration of organic solvent is greater than about $70 \%$. Although the column provides superior chromatographic properties and supports a broader variety of applications compared to conventional diol columns ${ }^{[3]}$, the available literature is yet devoid of conclusive experimental or theoretical evidences on how exactly retention is generated on this polar packing. This is the reason why authors decided to investigate the Acclaim column properties and behavior.

The retention mechanism of the HILIC phases makes them especially useful for separation of different polar organic compounds as a complement to RPLC. The aim of this study was to examine different test solutes retention/adsorption behavior (including analytes with different polarity, hydrophobicity and other physicochemical properties) on Acclaim Mixed Mode HILIC-1 column in methanol-water systems with high (RPLC mode) and low (HILIC mode) water content. In a HILIC technique the most popular organic component of a mobile phase is acetonitrile. Methanol is often used as an organic component of a mobile phase in RPLC and there are many papers presenting different investigations of retention process. Opposite to RPLC, the retention mechanism of the HILIC mode using alcohol as an eluent component was rather poorly explored and relatively few papers concerning the study on retention/sorption mechanism in such systems (also on diol adsorbents) can be found (e.g. [2]). Therefore authors performed a study on the presented above stationary phase using methanol-water mobile phase systems. Such studies seem relevant from a HILIC mechanism recognition point of view and constitute a certain aspect of novelty in such type analyses. Besides, in order to obtain insights into retention mechanism, for the same set of test solutes and for the wide range of mobile phase compositions, the influence of column temperature (using van't Hoff plots) was also established and discussed.

\section{Experimental}

The investigations were conducted using Dionex/Thermo Scientific Acclaim ${ }^{\circledR}$ Mixed-Mode HILIC-1 column (150 mm $\times 4.6 \mathrm{~mm}, 5 \mu \mathrm{m}$ particle size, $120 \AA$ pore size and $300 \mathrm{~m}^{2} / \mathrm{g}$ surface area). The hold-up volume of the column was derived from pycnometric measurements ${ }^{[4]}$ made at $20^{\circ} \mathrm{C}$ under atmospheric pressure using acetonitrile and trichloromethane as solvents. The densities of these two solvents at $20^{\circ} \mathrm{C}$ are 0.786 , and $1.48 \mathrm{~g} / \mathrm{cm}^{3}$, respectively. The column hold-up volume was established as $V_{0}=1.820 \pm 0.008 \mathrm{~cm}^{3}$ and the obtained values of total porosity, $\varepsilon_{t}$, and phase ratio, $\Phi$, are: 0.73 , and 0.3697 , respectively. The Primaide Merck-Hitachi (Germany/Japan) liquid chromatography (LC) system consisted of pump (model 1110), UV detector (model 1410), column oven (model 1310) and solvent degasser (model L-7612). 1,3,5-tri-tertbutylbenzene (TTBB), n-pentylbenzene (nPB), quercetin (Q), chrysin (CH), phenol (PH), 4-tert-butylphenol (TBP) and caffeine (CAF) were studied as test analytes using aqueous-methanol (MeOH) mobile phases both in RPLC (high content of water) and HILIC (high content of $\mathrm{MeOH}$ ) modes. Neither supporting salts nor buffers were added to the eluent so these compounds can only interact with the two eluent components for sorption onto the diol stationary phase. An ultrasonic bath 
was used to degas the mobile phases for 5 min just after mixing $\mathrm{MeOH}$ and water. In most of the studies, the content of aqueous phase in eluent was changed in the range of volume fraction, $\varphi$, from 0 to $0.6 \mathrm{~V} / \mathrm{V}$. For the detailed mobile phase compositions the reader is referred to the respective sections in the text as well as to figure legends and table captions. The flavonoids $(\mathrm{Q}, \mathrm{CH})$ were received from Sigma-Aldrich (Germany). The other substances and chromatographic grade solvents were purchased from Merck (Germany). Table 1 gives selected properties (molar mass, M, partition coefficient, $\log P_{o / w}$, numbers of of H-bond donors and acceptors) of test solutes analyzed in this study. Distilled, deionized and demineralized water was prepared on SolPure-78Z (ELKAR, Poland) water deionizer.

Table 1. Test solutes and their selected properties

\begin{tabular}{|c|c|c|c|c|c|c|}
\hline No. & Compound & Abbreviation & $M \mathrm{~g} / \mathrm{mol}$ & $\log P_{o / w}$ & $H$-bond donors & $H$-bond acceptors \\
\hline 1 & 1,3,5-tri-tert-butylbenzene & TTBB & 246 & 6.61 & - & - \\
\hline 2 & n-pentylbenzene & nPB & 148 & 4.26 & - & - \\
\hline 3 & quercetin & Q & 302 & 2.16 & 5 & 7 \\
\hline 4 & chrysin & $\mathrm{CH}$ & 254 & 3.01 & 2 & 4 \\
\hline 5 & phenol & $\mathrm{PH}$ & 94 & 1.67 & 1 & 1 \\
\hline 6 & 4-tert-butylphenol & TBP & 150 & 3.21 & 1 & 1 \\
\hline 7 & caffeine & CAF & 194 & -0.55 & - & 3 \\
\hline
\end{tabular}

All investigations were carried out isocratically at $1.0 \mathrm{~cm}^{3} / \mathrm{min}$ flow rate. The injection volume (samples dissolved in the mobile phase) was $0.02 \mathrm{~cm}^{3}$ of working standard solutions $\left(20 \mu \mathrm{g} / \mathrm{cm}^{3}\right)$. At the beginning of each set of experiments, the column was equilibrated at adequate temperature by washing with approximately 20 column hold-up volumes, $V_{0}$, of the fresh mobile phase. The retention times, $t_{r}$, of sample compounds were measured in triplicate for mobile phases containing various volume ratios of methanol:water at $20,25,30,35,40,45$ and $50^{\circ} \mathrm{C}$. The experimental data (the retention times for the peak apexes) were corrected for the extra-column volume dead time (i.e., the time needed for the eluent to flow through the capillaries connecting the valve with the column and the column with the detector), $t_{d}=0.08 \mathrm{~min}$. On the basis of the experimental $t_{r}$, the retention factors, $k=\left(V_{r}-V_{0}\right) / V_{0}=\left(t_{r}-t_{0}\right) / t_{0}$, were calculated and arithmetic means from three repeated experiments were used in the regression analysis for the determination of thermodynamic constants in the van't Hoff equation. The linear regression analysis was conducted using OriginPro 8 SRO software.

\section{Results and discussion}

\subsection{Solute retention in RPLC and HILIC modes}

Aromatic hydrocarbons (TTBB, nPB), flavonoids (Q, CH) and phenols (PH, TBP) exhibit similar, nonlinear shapes of $\ln k=f\left(\varphi_{\mathrm{H}_{2} \mathrm{O}}\right)$ dependencies in the analyzed systems-see Figure 1A. Generally, regardless of temperature, these substances are very poorly, however noticeably retained in systems with water content $0-25 \% \mathrm{~V} / \mathrm{V}$ (HILIC mode). After exceeding approximately of the $25 \%$ water content in MeOH (RPLC mode), the retention factors of these substances are significantly higher. Comparing the retention of the test substances belonging to the respective types of compounds it is also apparent that the more hydrophobic compounds (Table 1) were more strongly retained than the less hydrophobic in the whole analyzed range of eluent compositions and temperatures. For example, as it can be seen in Figure 1A, at column temperature $20^{\circ} \mathrm{C}$ the more hydrophobic chrysin $\left(\log P_{o / w}=3.01\right)$ was strongly retained than the less hydrophobic quercetin $\left(\log P_{o / w}=2.16\right)$. The same phenomenon was observed in the whole range of analyzed temperatures.

For CAF studied in aqueous-methanol mobile phases, U-shape plots of $\ln k$ versus the water phase concentration were observed in the whole temperature range-see Figure 1B. Besides, caffeine exhibits relatively low retention in HILIC and RPLC modes.

To evaluate the impact of mobile phase composition on retention, as suggested by Hemsröm and Irgum ${ }^{[5]}$, in Figures 1C and 1D a series of $\ln k$ versus $\ln \left(\varphi_{\mathrm{H}_{2} \mathrm{O}}\right)$ dependencies were also reported. In theory, the clear linearity of $\ln k=f$ $\left(\varphi_{\mathrm{H}_{2} \mathrm{O}}\right)$ would indicate predominance of a partitioning process, whereas the linearity of $\ln k=f\left(\ln \varphi_{\mathrm{H}_{2} \mathrm{O}}\right)$ would reveal an adsorption mechanism ${ }^{[5]}$. As shown in Figure 1, no linearity was observed in the both cases. The same situation occurs in the whole considered temperature range. This may be an evidence of relatively complex retention mechanism in the analyzed systems.

\subsection{Effect of column temperature-van't Hoff plots}

Column temperature is also an important parameter that affects the retention of compounds in liquid chromatography. 
The main reason for studying the relationship between solute retention factors and temperature was to gain insights into changes of retention mechanism involving solutes of different properties and diol-stationary phase used both in the HILIC and the RPLC modes and in systems with different content of $\mathrm{MeOH}$ as an organic component of the mobile phase. The relationship between retention factor, $k$, and column temperature, $T$, is often described by van't Hoff equation ${ }^{[6]}$ :

$$
\ln k=-\frac{\Delta H^{o}}{R \cdot T}+\frac{\Delta \Sigma^{o}}{R}+\ln \Phi
$$
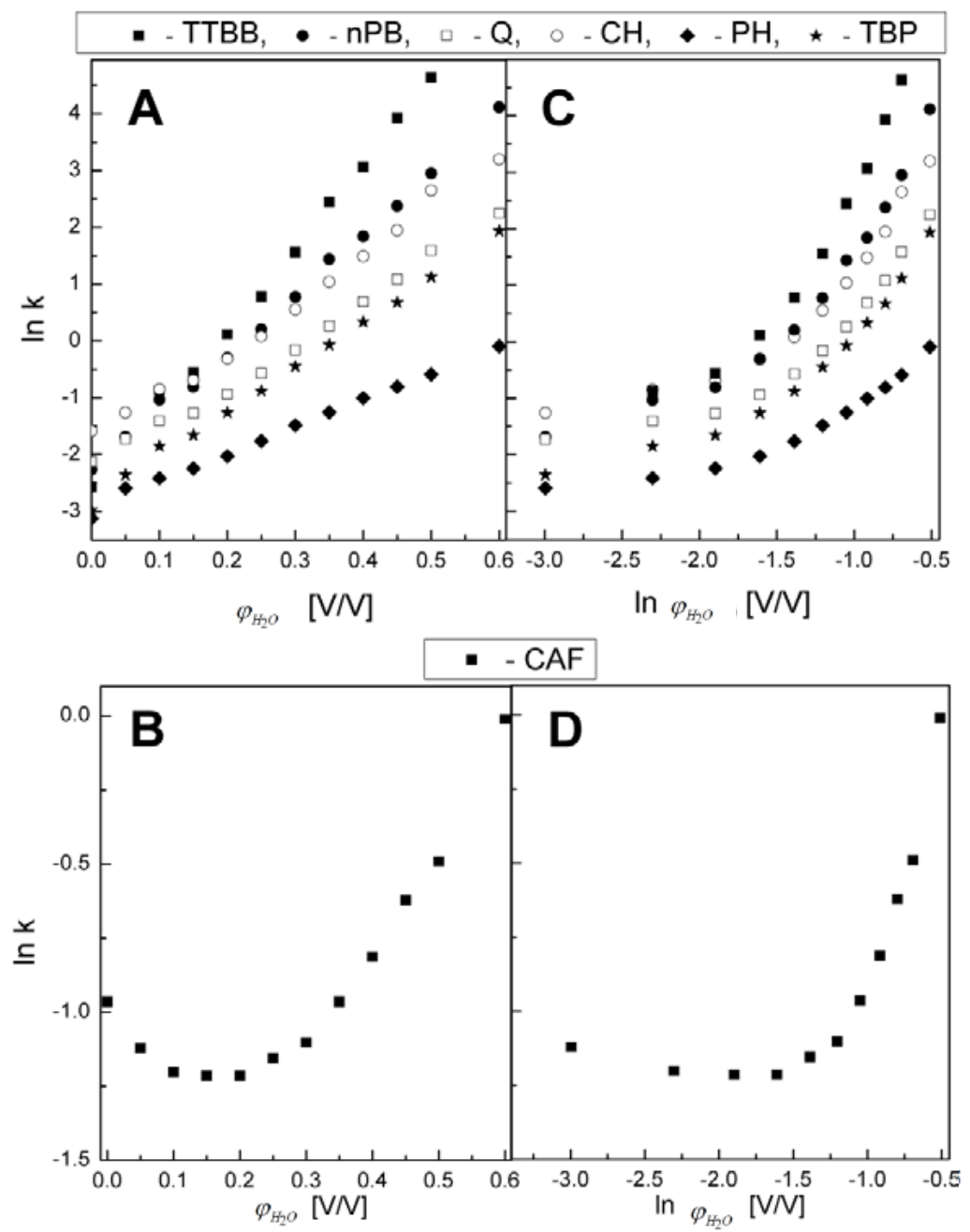

Figure 1. Plots of $\ln k$ versus $\varphi_{\mathrm{H}_{2} \mathrm{O}}$ and $\ln k$ versus $\ln \left(\varphi_{\mathrm{H}_{2} \mathrm{o}}\right)$ considering seven model compounds at column temperature $20^{\circ} \mathrm{C}$

In Figures 2-5, the experimental $\ln k$ versus $1 / T$ plots (points) and best fitted regression results obtained for van't Hoff equation (lines) for all test solutes, applied mobile phase compositions, and temperature range $20-50^{\circ} \mathrm{C}$ were presented. Note, that higher temperature values were not studied due to instability of the column according to the manufacturer's recommendation. Table 2 lists the regression statistics of eq. (1) for systems presented in Figures 2-5. Generally, the results of investigations show that the retention of all analyzed test substances decreased at higher temperatures, tantamount to negative retention enthalpies (Table 2) indicating process of exothermic type. The same trend was observed for all mobile phase compositions. The experimental $\ln k=f(1 / T)$ plots indicate, that linear, slightly nonlinear and also nonlinear dependencies were observed in the analyzed systems. This is confirmed by listed values of correlation coefficients $R^{2}$ in Table 2, describing the validity of the van't Hoff model (eq. 1).

In the case of TTBB (Figure 2A) and nPB (Figure 2B) studied in systems containing 5-25\% V/V and 40-50\% (40$60 \%$ for $\mathrm{nPB}$ ) V/V water, $R^{2}$ values close to 1 (Table 2 ) demonstrate the validity of eq. (1) and suggest that mainly single retention mechanism controls their retention over the experimental temperature range. Curvatures of the van't Hoff regression plots were revealed in mobile phase with pure $\mathrm{MeOH}\left(R^{2}\right.$ close to 0.9$)$, but even more significantly $\left(R^{2}<0.8\right)$ 
at concentrations of water 35\% V/V. For Q (Figure 3A) and CH (Figure 3B) highly linear van't Hoff plots ( $R^{2}$ close to 0.99) were observed with eluents containing 5-30\% and 40-60\% V/V water (Table 2), suggesting dominance of a single retention mechanism over the experimental temperature range. Lower values of $R^{2}$ occur in the case of mobile phase with pure $\mathrm{MeOH}$ ( $R^{2}$ close to $0.94-0.96$ for both substances) and at concentrations of water $35 \% \mathrm{~V} / \mathrm{V}$ ( $R^{2}$ for both substances are close to 0.93-0.95). For PH (Figure 4A) the regression results for eq. (1) with $R^{2}$ close to 0.99 were obtained for eluents containing 5\%,15\%,25\% and $60 \% \mathrm{~V} / \mathrm{V}$ water. The correlation coefficients are lower than 0.96 (especially for $\varphi_{\mathrm{H}_{2} \mathrm{O}}$ $=10 \%, R^{2}=0.8652$ ) for other eluent compositions (Table 2). For TBP (Figure 4B) the $R^{2}$ close to 0.99 were observed only in $15 \%, 25 \%, 50 \%$ and $60 \% \mathrm{~V} / \mathrm{V}$ water (Table 2). The $R^{2}$ lower than 0.98 (especially for $\varphi_{\mathrm{H}_{2} \mathrm{O}}=0 \%$ and $35 \%, R^{2}$ are equal 0.9016 , and 0.8757 respectively) were obtained for other eluent compositions. The experimental retentiontemperature dependences exhibit linearity for CAF (Figure 5) in mobile phases with water content range 0-25\% V/V. $R^{2}$ values are rather stable and greater than 0.99 . This suggests that mainly single retention mechanism controls the retention in the examined eluents and temperatures. Slight deviations from linearity of $\ln k=f(1 / T)$ were revealed for the water content range in mobile phase $30-50 \% \mathrm{~V} / \mathrm{V}$ (see Table 2)-the minimal value of $R^{2}=0.9647$ was obtained for $\varphi_{H_{2} O}=50 \%$ $\mathrm{V} / \mathrm{V}$ whereupon $R^{2}$ was increased up to 0.9898 for $\varphi_{\mathrm{H}_{2} \mathrm{O}}=60 \% \mathrm{~V} / \mathrm{V}$. When analyzing the and the values determined for individual compounds, a significant variability of these parameters (especially for flavonoids and phenols) depending the mobile phase composition and compound's hydrophobicity was observed (Table 2). For example the values for more hydrophobic $\mathrm{CH}$ are higher than for less hydrophobic Q when eluents containing from 0 to $30 \% \mathrm{~V} / \mathrm{V}$ of water (Table 2) were applied. For the rest of eluents the phenomenon is opposite. At the same time the values for $\mathrm{CH}$ are greater than the values for $\mathrm{Q}$ in the whole range of eluent compositions. Similar relationship was also observed for PH and TBP-see Table 2. The other picture of the enthalpy and entropy variability depending on the mobile phase composition is observed for aromatic hydrocarbons and acids. The values for less hydrophobic nPB are higher than for more hydrophobic TTBB (Table 2) for all investigated mobile phase compositions, while the values are generally higher for TTBB except for eluents with low water content $\left(\varphi_{\mathrm{H}_{2} \mathrm{O}}\right.$ range $\left.0-10 \% \mathrm{~V} / \mathrm{V}\right)$.

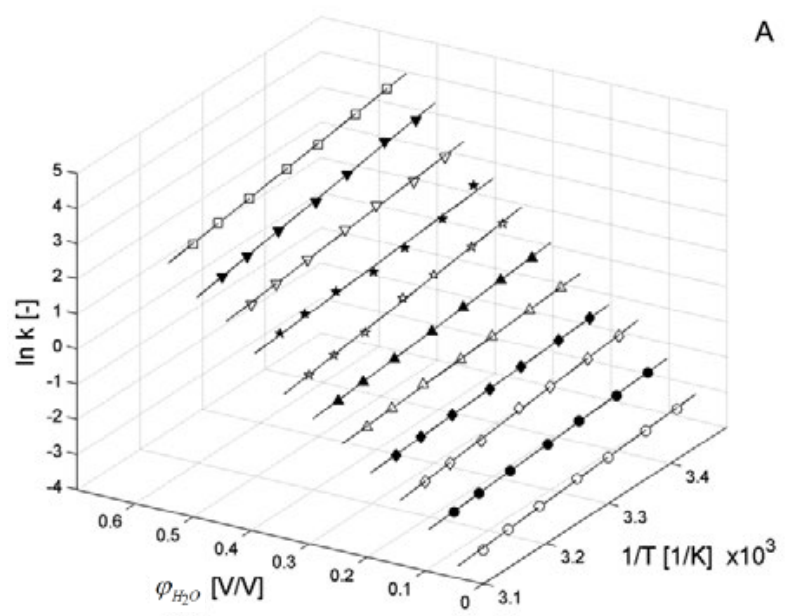

A

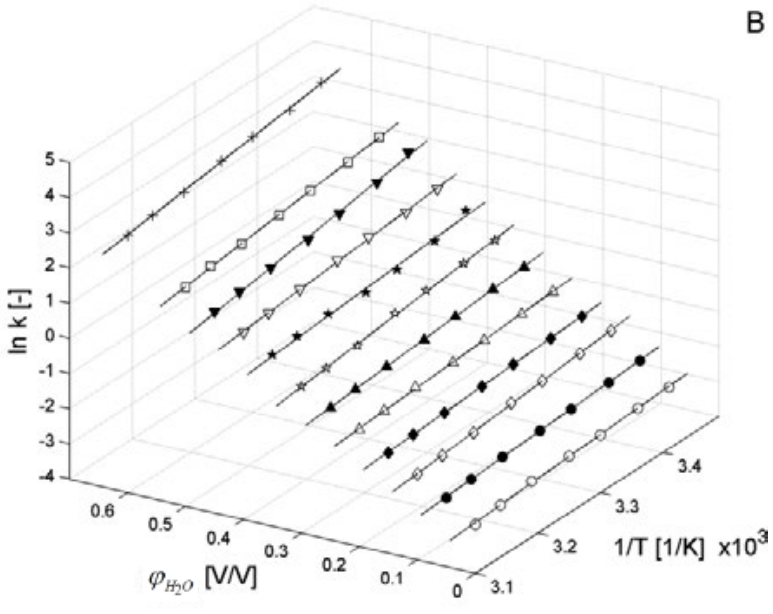

Figure 2. The van't Hoff plots of TTBB (A) and nPB (B) for different mobile phase compositions. Points-experimental data, lines-van't Hoff model 

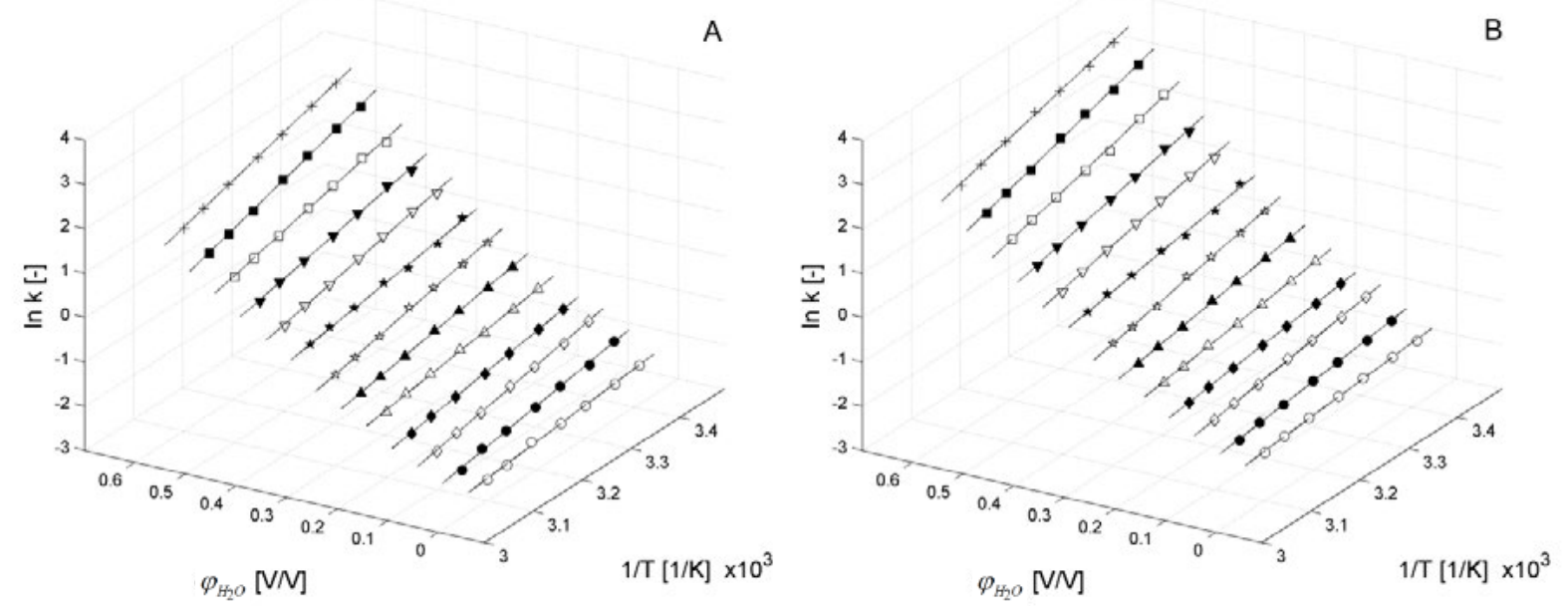

Figure 3. The van't Hoff plots of Q (A) and CH (B) for different mobile phase compositions. Points-experimental data, lines-van't Hoff model

Table 2. Standard enthalpy $\Delta H^{0}[\mathrm{~kJ} / \mathrm{mol}]$ and standard entropy $\Delta S^{0}[\mathrm{~J} / \mathrm{mol} \cdot \mathrm{K}]$ changes of solute transfer (regression results from van't Hoff equation) for the analyzed chromatographic systems

\begin{tabular}{cccccccccccccccc}
\hline $\begin{array}{c}\varphi_{\mathrm{H}_{2} \mathrm{O}} \\
\mathrm{V} / \mathrm{V}\end{array}$ & $\Delta H^{0}$ & $\begin{array}{c}\Delta S^{0} \\
\mathrm{TTBB}\end{array}$ & $\Delta R^{2}$ & $\Delta H^{0}$ & $\begin{array}{c}\Delta S^{0} \\
\mathrm{nPB}\end{array}$ & $\Delta R^{2}$ & $\Delta H^{0}$ & $\Delta S^{0}$ & $\Delta R^{2}$ & $\Delta H^{0}$ & $\Delta S^{0}$ & $\Delta R^{2}$ & $\Delta H^{0}$ & $\Delta S^{0}$ & $\Delta R^{2}$ \\
$\mathrm{PH}$ & \\
\hline 0 & -11.81 & -61.37 & 0.9499 & -8.98 & -50.18 & 0.9329 & -9.74 & -42.65 & 0.9403 & -8.74 & -34.42 & 0.9643 & -20.41 & -94.85 & 0.9401 \\
0.05 & -11.05 & -51.96 & 0.9957 & -9.23 & -46.45 & 0.9951 & -16.96 & -64.07 & 0.9816 & -12.80 & -45.79 & 0.9918 & -22.10 & -97.16 & 0.9841 \\
0.1 & -16.81 & -64.39 & 0.9687 & -14.41 & -58.65 & 0.9873 & -19.23 & -68.94 & 0.9930 & -15.51 & -51.68 & 0.9922 & -17.48 & -79.22 & 0.8652 \\
0.15 & -9.27 & -36.27 & 0.9985 & -8.80 & -37.73 & 0.9989 & -15.91 & -56.52 & 0.9992 & -12.86 & -41.36 & 0.9996 & -13.29 & -64.03 & 0.9898 \\
0.2 & -10.69 & -35.44 & 0.9865 & -9.68 & -36.42 & 0.9864 & -14.65 & -49.43 & 0.9833 & -13.99 & -42.09 & 0.9973 & -8.32 & -45.27 & 0.9423 \\
0.25 & -14.49 & -42.76 & 0.9936 & -12.63 & -42.22 & 0.9966 & -17.62 & -56.48 & 0.9947 & -16.99 & -48.93 & 0.9915 & -12.09 & -55.78 & 0.9958 \\
0.3 & -21.39 & -59.69 & 0.9660 & -17.54 & -61.11 & 0.9722 & -21.38 & -65.75 & 0.9854 & -21.72 & -61.05 & 0.9827 & -13.42 & -57.97 & 0.9760 \\
0.35 & -13.36 & -26.93 & 0.6730 & -12.12 & -31.70 & 0.7515 & -15.73 & -43.70 & 0.9505 & -16.27 & -39.33 & 0.9336 & -8.09 & -38.29 & 0.9459 \\
0.4 & -16.96 & -32.09 & 0.9994 & -14.16 & -33.71 & 0.9962 & -21.54 & -58.98 & 0.9841 & -22.69 & -56.19 & 0.9821 & -10.26 & -43.15 & 0.9690 \\
0.45 & -25.68 & -54.60 & 0.9852 & -24.90 & -64.36 & 0.9714 & -21.43 & -55.27 & 0.9795 & -23.24 & -54.20 & 0.9848 & -12.54 & -49.23 & 0.9575 \\
0.5 & -22.87 & -39.61 & 0.9989 & -18.86 & -40.95 & 0.9960 & -24.27 & $-66,62$ & 0.9795 & -27.82 & -64.81 & 0.9895 & -11.86 & -45.22 & 0.9770 \\
0.55 & - & - & - & - & - & - & -30.60 & -77.03 & 0.9912 & -30.90 & -70.32 & 0.9884 & - & - & - \\
0.6 & - & - & - & -20.55 & -37.15 & 0.9860 & -28.45 & -66.67 & 0.9990 & -27.02 & -53.97 & 0.9903 & -11.64 & -40.68 & 0.9885 \\
\hline
\end{tabular}

continued Table 2

\begin{tabular}{ccccccc}
\multicolumn{7}{c}{ continued Table 2 } \\
\hline$\varphi_{H_{2} O}$ & $\Delta H^{0}$ & $\Delta S^{0}$ & $\Delta R^{2}$ & $\Delta H^{0}$ & $\Delta S^{0}$ & $\Delta R^{2}$ \\
$\mathrm{~V} / \mathrm{V}$ & & $\mathrm{TBP}$ & & & $\mathrm{CAF}$ & \\
\hline 0 & -17.51 & -83.60 & 0.9016 & -13.35 & -53.59 & 0.9946 \\
0.05 & -14.19 & -67.89 & 0.9575 & -11.65 & -49.01 & 0.9916 \\
0.1 & -15.81 & -69.15 & 0.9723 & -10.80 & -46.83 & 0.9977 \\
0.15 & -10.64 & -50.01 & 0.9964 & -11.99 & -50.89 & 0.9952 \\
0.2 & -9.62 & -43.22 & 0.9748 & -10.51 & -45.94 & 0.9920 \\
0.25 & -11.74 & -47.20 & 0.9891 & -11.71 & -49.54 & 0.9969 \\
0.3 & -15.06 & -54.88 & 0.9698 & -12.56 & -51.86 & 0.9896 \\
0.35 & -8.09 & -28.62 & 0.8757 & -10.41 & -43.73 & 0.9883 \\
0.4 & -13.33 & -42.19 & 0.9631 & -11.46 & -45.81 & 0.9908 \\
0.45 & -13.38 & -39.88 & 0.9819 & -15.47 & -57.89 & 0.9876 \\
0.5 & -14.57 & -40.42 & 0.9940 & -14.05 & -51.98 & 0.9647 \\
0.6 & -14.91 & -34.85 & 0.9907 & -13.66 & -46.97 & 0.9898 \\
\hline
\end{tabular}



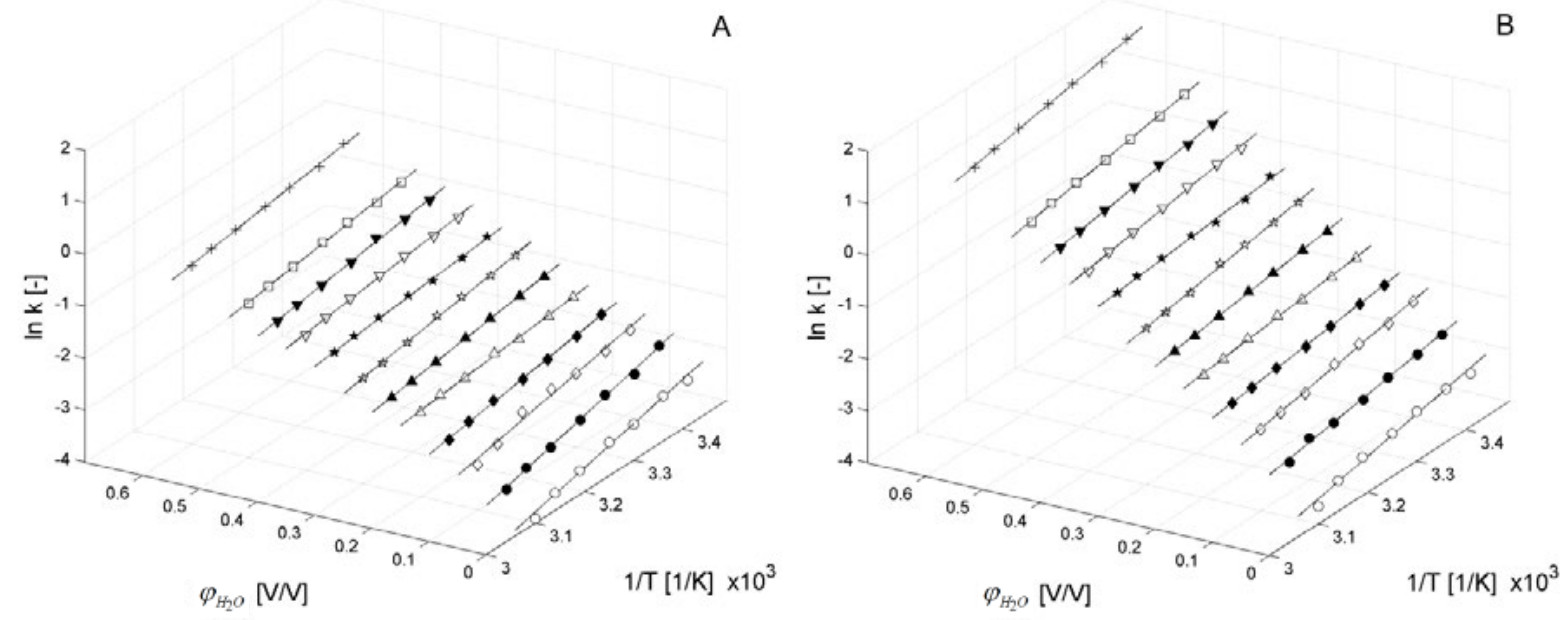

Figure 4. Van't Hoff plots of PH (A) and TBP (B) for different mobile phase compositions. Points-experimental data, lines-van't Hoff model

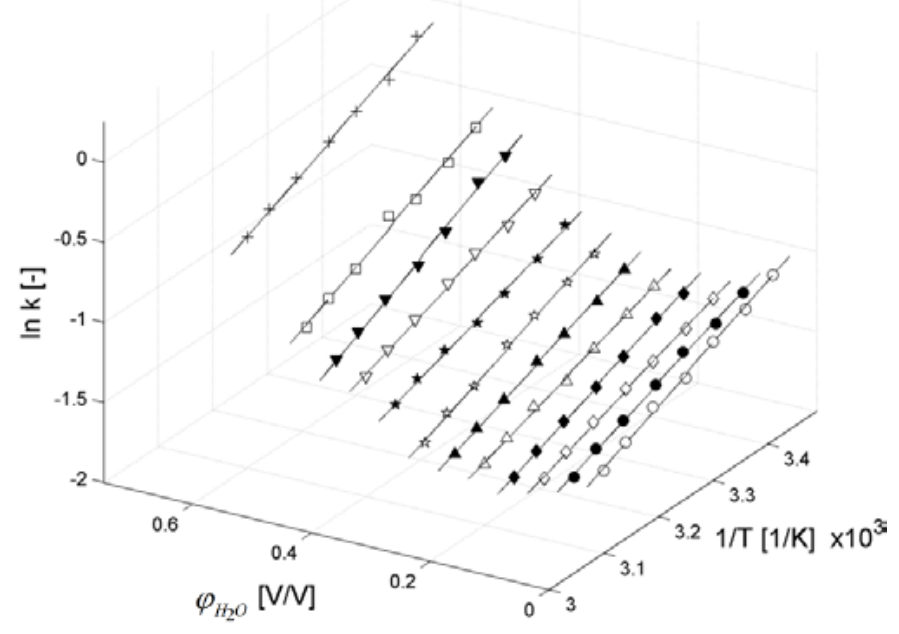

Figure 5. Van't Hoff plots of CAF for different mobile phase compositions. Points-experimental data, lines-van't Hoff model

As previously mentioned, in accordance to Hemsröm and $\operatorname{Irgum}^{[5]}$, nonlinear $\ln k=f\left(\varphi_{\mathrm{H}_{2} \mathrm{O}}\right)$ and $\ln k=f(\ln$ $\varphi_{\mathrm{H}_{2} \mathrm{O}}$ ) dependencies (Figure 1) suggest that the retention mechanism is rather complex, i.e. surface adsorption (via different sorption interactions type) may coexist in different proportions with a partition mechanism (mostly driven by hydrophobicity). Due to presence of long-chain alkyl groups (which are capable of separating different molecules via hydrophobic interaction) on the adsorbent surface, the hydrophobic interactions may influence to some extent the global retention mechanism of the tested compounds. It is especially evident for the non-polar aromatic hydrocarbons tested in this study (where the retention mechanism is inherent to the RPLC). However, taking into account the presented results as well as Jaroniec-Martire theory ${ }^{[7]}$, the presence of mixed adsorption-partition mechanism is in this case also possible.

As it was shown in the previous section, the retention behavior of the examined substances strongly depends on their hydrophobicity. On the other hand, when multiple polar functional groups such as e.g. $-\mathrm{OH},-\mathrm{NH}_{2}$ contribute to analyte polarity, hydrogen-bonding interactions could affect the global retention mechanism in most HILIC columns ${ }^{[8]}$. Flavonoids, phenols and caffeine have a noticeable hydrogen-bonding potential sites (Table 1) and (as shown previously) are rather poorly retained in the HILIC mode presumably due to the presence of methanol in the mobile phase. It is commonly accepted that in the HILIC mode a water-enriched liquid layer is established within the stationary phase ${ }^{[1]}$, thus partitioning of the solutes from the mobile phase into the hydrophilic layer occurs. Methanol as an organic component of the mobile phase with its strong ability for hydrogen bonding (this polar protic solvent can be both donor and acceptor of hydrogen bonds) may disturb the formation of the water layer at the stationary phase surface, replacing water molecules, thus producing a more hydrophobic stationary phase ${ }^{[1,8]}$. As a consequence, the analytes with hydrogen-bonding potentials are less retained. It should be also noticed, that replacing $\mathrm{MeOH}$ with acetonitrile (this aprotic solvent can be only hydrogen 
bond acceptor) as a weak eluent component gives in HILIC mode higher retention factors e.g. for CAF ${ }^{[9]}$. Basing on the above observations it is clearly evident that the eluting strength is also correlated with the different hydrogen bonding potentials of the organic solvents. Thus, except for the hydrophobic retention, the hydrogen bonding interactions (especially in the HILIC mode) could also influence the retention behavior of flavonoids, phenols and caffeine in the analyzed mobile phases.

Regarding polar columns, many strongly or moderately polar compounds show U-shape plots of $k$ versus the concentration of water in the mobile phase, with a minimum corresponding to the transition point from the RPLC to the HILIC mechanism ${ }^{[1]}$. In this work, such dependence was observed for caffeine. As it was mentioned above, the HILIC retention is controlled by liquid-liquid partitioning between the mobile phase and the water-enriched solvent layer at the surface of the polar stationary phase ${ }^{[1]}$. Hydrophobic, hydrogen bonding and other electrostatic-type (e.g. ion-exchange or ion repulsion) interactions may also contribute to the retention, depending on the sample, the stationary phase and the composition of the mobile phase ${ }^{[8,10]}$. Opposite to the HILIC, the RPLC mode retention is essentially controlled by nonpolar (solvophobic) interactions ${ }^{[1]}$. Simultaneously, in the RPLC mode interactions with polar groups and residual silanols in the stationary phase may affect the retention. On the other hand, solvophobic interactions may contribute to some extent to the retention under the HILIC conditions, as most polar stationary phases (except for bare silica) contain some nonpolar structural elements, such as hydrocarbon "spacers" enabling covalent attachment of polar groups to the surface of the silica support. Hence, depending on the amount of the organic in the mobile phase, these combined effects may control the resultant retention in many HILIC columns. In the presence of the combined HILIC/RPLC retention mechanism, the RPLC behavior predominates in water-rich mobile phases and the retention decreases with rising concentration of the organic solvent, until minimum retention is achieved. The retention starts to rise again with further increase of the organic solvent concentration, as the polar interactions become stronger than the solvophobic ones in highly organic mobile phases. $\mathrm{CAF}$ is the most hydrophilic substance among being analyzed and has a certain hydrogen-bonding potential (Table 1). So, the retention behavior of CAF under HILIC conditions is presumably connected with hydrophilic partition in which (due to the rather low $\mathrm{CAF}$ retention and presence of $\mathrm{MeOH}$ in mobile phase) H-bond interactions may also affect the retention mechanism. At the same time, CAF is rather bulky molecule (M=194 g/mol), which may be retained in water-rich eluents by hydrophobic interactions and connected with the increase of CAF retention observed in the RPLC mode.

The change in retention as a function of the column temperature is dependent on both functional groups of an analyte and a stationary phase as well as on a composition of a mobile phase. The presented in previous section results of investigations indicate that the retention process is exothermic in all analyzed chromatographic systems. The observed linearity or non-linearity of van't Hoff plots provides information on whether the retention mechanism(s) changes over the studied temperature range or not ${ }^{[11]}$. The obtained in some of the analyzed systems non-linear plots could indicate: (a) alteration in the main retention mechanism to another with increasing temperature (e.g. the electrostatic interactions are diminished, while the adsorptive forces become prevalent); (b) some conformation changes in a bonded phase or (c) variation of the thickness of adsorbed part of the mobile phase (e.g. water) on the stationary phases with temperature. In addition, the above discussed mechanisms may be combined ${ }^{[12]}$. Noteworthy is also the fact that the enthalpy values derived for individual test substances were very different depending on the mobile phase composition that could also indicate the existence of strong specific (secondary) interactions between these substances and functional groups of the tested stationary phase. Taking into account two facts: (i) the surface of actual adsorbents is heterogeneous by nature and (ii) the sample components have multiple functional groups, the observed nonlinearities of van't Hoff plots may be also caused by the specific analyte-stationary phase interactions connected with the presence of energetically different active sites on the stationary phase surface (both alkyl groups with polar terminus and residual silanols can act as low- and/or highenergy active sites). The presence of such interactions was confirmed in literature ${ }^{[13]}$ for many silica based (and not only) adsorbents, mobile phases and chromatographed compounds. Besides, in accordance to Kim ${ }^{[14]}$, qualitative assessments of the adsorption energy distribution (AED) at different temperatures indicate that temperature has a significant influence on the adsorption energy on the high-energy sites and on the saturation capacity of the low-energy sites. The previous investigations of $\mathrm{AED}^{[15]}$ conducted in the same column for $\mathrm{CAF}$ in different $\mathrm{MeOH}$-water systems, indicate that in the RPLC mode the adsorption mechanism is seriously heterogeneous. In contrast, in the HILIC mode where the van't Hoff plots are almost linear, the adsorption behavior is quasi-homogeneous. It was also stated, that e.g. phenols (PH, TBP) exhibit heterogeneous adsorption behavior both in the HILIC and the RPLC modes (it is especially evident in systems where nonlinearities in van't Hoff plots were observed). Thus it seems, that the surface heterogeneity could in some cases effect the retention mechanism in the tested column and provide explanation (to a certain degree) for the observed nonlinearities in van't Hoff plots in some of the analyzed systems. 
It should be also pointed that, if the observed nonlinearities in the van't Hoff plots were due to changes in the enthalpy and the entropy of either solutes, but not caused by a temperature-dependent phase ratio, the obtained results may be the evidence of a complex retention mechanism in the analyzed systems. Because authors were focused only on practical observations of selected test substances retention behavior, therefore investigations related to the phase ratio variability were beyond the scope of the present study.

\section{Conclusions}

In this study the retention behavior of several test substances on Acclaim Mixed Mode HILIC-1 column was analyzed using $\mathrm{MeOH}$ as weak eluent component. The acquisition of retention data for chemically different compounds in a wide range of temperatures and mobile phase compositions allowed evaluation of their retention mechanism(s) variability in the tested column. Although the column with structurally simple ligand was chosen, rather complex processes (that generate retention for chemically different compounds in different mobile phase compositions) were revealed. It was found that depending on mobile phase composition, both linear, slightly curved and curved van't Hoff plots were observed in the studied systems. This leads to the conclusion that a single retention mechanism may dominate in some systems and simultaneously in others the presence of additional mechanisms is highly probable. Moreover, fairly significant changes of the and the values were observed for all tested compounds depending on the mobile phase composition, that may indicate the presence of specific interactions between the analytes and functional groups of the tested stationary phase. It was also found that the retention behavior strongly depends on compounds hydrophobicity in the analyzed systems. On the other hand, in case of the compounds with hydrogen-bonding potentials, the presence of $\mathrm{MeOH}$ in eluent may cause the $\mathrm{H}$-bond interactions also contribute to the retention mechanism. Additionally, the presence of other specific interactions connected with energetic heterogeneity of stationary phase surface should not be ruled out.

Summing up, the presented results confirm that the tested column is suitable for both RPLC (in highly aqueous conditions) and HILIC (in highly organic conditions) applications.

\section{Compliance with ethical standards}

The authors declare that they have no conflict of interest.

No research involving human participants and/or animals was included in the study.

\section{Funding}

This research did not receive any specific grant from funding agencies in the public, commercial, or not-for-profit sectors.

\section{References}

[1] Jandera P. Stationary and mobile phases in hydrophilic interaction chromatography: a review. Anal. Chim. Acta. 2011; 692: 1-25. Available from: doi: 10.1016/j.aca.2011.02.047.

[2] Liu M., Ostovic J., Chen E. X., Cauchon N. Hydrophilic interaction liquid chromatography with alcohol as a weak eluent. J. Chrom. A. 2009; 1216: 2362-2370. Available from: doi: 10.1016/j.chroma.2009.01.012.

[3] Liu X., Pohl C. New hydrophilic interaction/reversed-phase mixed-mode stationary phase and its application for analysis of non-ionic ethoxylated surfactants. J. Chrom. A. 2008; 1191: 83-89. Available from: doi: 10.1016/ j.chroma.2007.12.012.

[4] McCormick R.M., Karger B.L. Distribution Phenomena of Mobile-Phase Components and Determination of Dead Volume in Reversed-Phase Liquid Chromatography. Anal. Chem. 1980; 52: 2249-2257. Available from: doi: 10.1021/ ac50064a005.

[5] Hemsröm P., Irgum K. Hydrophilic interaction chromatography. J. Sep. Sci. 2006; 29: 1784-1821. Available from: doi: $10.1002 /$ jssc.200600199.

[6] Tchapla A., Heron S., Cohn H., Guiochon G. Role of temperature in the behavior of a homologous series in reversed phase liquid chromatography. Anal. Chem. 1988; 60: 1443-1448. Available from: doi: 10.1021/ac00165a019.

[7] Jaroniec M. Partition and displacement models in reversed-phase liquid chromatography with mixed eluents - Review Article. J. Chrom. A. 1993; 656: 37-50. Available from: doi: 10.1016/0021-9673(93)80796-B.

[8] Hao Z., Xiao B., Wang N. Impact of column temperature and mobile phase components on selectivity of hydrophilic interaction chromatography (HILIC). J. Sep. Sci. 2008; 31: 1449-1464. Available from: doi: 10.1002/jssc.200700624. 
[9] Kamińska J., Zapała W. Influence of modifier concentration on retention in hydrophilic interaction chromatography preliminary investigations. Chem. Eng. Equip. 2015; 54(1): 4-7.

[10] Wu J.Y., Bicker W., Lindner W. Separation properties of novel and commercial polar stationary phases in hydrophilic interaction and reversed-phase liquid chromatography mode. J. Sep. Sci. 2008; 31: 1492-1503. Available from: doi: $10.1002 /$ jssc.200800017.

[11] Melander W., Campbell D.E., Horvath C. Enthalpy-entropy compensation in reversed-phase chromatography. $J$. Chrom. 1978; 158: 215-225. Available from: doi: 10.1016/S00219673(00)89968-9.

[12] Kucera R.P. Kovarikova P., Klivicky M., Klimes J. The retention behaviour of polar compounds on zirconia based stationary phases under hydrophilic interaction liquid chromatography conditions. J. Chrom. A. 2011; 1218: 69816986.

[13] Gritti F., Santos Pereira A., Sandra P., Guiochon G. Comparison of the adsorption mechanisms of pyridine in hydrophilic interaction chromatography and in reversed-phase aqueous liquid chromatography. J. Chrom. A. 2009; 1216: 8496-8504. Available from: doi: 10.1016/j.chroma.2009.10.009.

[14] Kim H., Gritti F., Guiochon G. Effect of the temperature on the isotherm parameters of phenol in reversed-phase liquid chromatography. J. Chrom. A. 2004; 1049: 25-36. Available from: doi: 10.1016/j.chroma.2004.08.025.

[15] Kamińska J., Chutkowski M., Zapała L., Zapała W. Studies on the sorption behavior of quercetin, phenol, and caffeine as test substances on diol mixed-mode HILIC column. $J L C \& R T$. 2016; 39(3): 161-170. Available from: doi: $10.1080 / 10826076.2016 .1141364$. 